\title{
Influência dos estilos de aprendizagem na utilização de ambientes virtuais de aprendizagem
}

\section{Influence of learning styles on the use of virtual learning environments}

\author{
Paulo Alves*, Luísa Miranda*, Daniela Melaré**, Carlos Morais,**** \\ *Instituto Politécnico de Bragança, **Universidade Aberta, ***Universidade do Minho
}

\begin{abstract}
Resumo
Este estudo aborda os estilos de aprendizagem dos estudantes de ensino superior e o uso de ferramentas digitais. O principal objetivo deste estudo foi averiguar a influência dos estilos de aprendizagem no acesso às ferramentas do ambiente virtual de aprendizagem (AVA). Os dados foram obtidos no AVA através do questionário CHAEA e dos registos de acesso às ferramentas armazenados automaticamente no AVA. Constatou-se que o estilo reflexivo apresenta a média mais alta das pontuações obtidas entre todos os estilos e que a ferramenta recursos apresenta o número mais elevado de acessos.

Palavras-chave: Estilos de aprendizagem, CHAEA, ensino superior, ambiente virtual de aprendizagem, acesso a ferramentas digitais.
\end{abstract}

\begin{abstract}
This study focuses on the issue involving higher education students' learning styles and the use of digital tools. The main aim of this research was to determine the influence that learning styles may have on the access to the tools of the virtual learning environment. The data related to learning styles was obtained through CHAEA questionnaire.

In the light of the results obtained, it was found that the reflective style was the one which showed the highest mean of the scores obtained concerning the subjects' learning styles, and that the tool which showed the highest number of accesses was the Resources.

Keywords: Learning styles, CHAEA, higher education, virtual learning environment, access to digital tools.
\end{abstract}

\section{Introdução}

Os recursos digitais e a sua utilização têm grande importância na vida das pessoas quando respondem aos seus interesses e necessidades e se adequam aos seus estilos de aprendizagem.

Para averiguar a influência dos estilos de aprendizagem no acesso aos recursos digitais de um ambiente virtual de aprendizagem (AVA), desenvolveu-se uma investigação numa instituição portuguesa de ensino superior que disponibiliza à sua comunidade académica um AVA, com vários recursos digitais de apoio às atividades desenvolvidas pelos seus membros e, com principal destaque para as atividades dos estudantes e dos docentes.
Esta investigação pode contribuir para obter indicadores que permitam melhorar o processo de ensino e aprendizagem dos estudantes, tendo em conta os seus estilos de aprendizagem e os recursos digitais disponibilizados nos AVA das instituições a que pertencem. Os principais objetivos desta investigação são:

- Caracterizar os estilos de aprendizagem de uma amostra de estudantes do ensino superior;

- Identificar, num semestre letivo, a frequência com que os estudantes acederam aos recursos digitais disponíveis no ambiente no AVA;

- Avaliar a relação entre frequência de acesso aos recursos digitais, disponíveis no AVA, e os estilos de aprendizagem dos estudantes.

$\mathrm{Na}$ identificação dos estilos de aprendizagem dos sujeitos da amostra utilizou-se o questionário "Cuestionario Honey-Alonso de Estilos de Aprendizaje (CHAEA)", e na determinação da frequência de acessos aos recursos digitais utilizou-se a base de dados associada ao AVA, onde esses registos são armazenados automaticamente.

Da organização do artigo sobressaem os tópicos: Introdução, fundamentação teórica, metodologia, apresentação e discussão dos resultados, e conclusões, terminando com as referências bibliográficas.

\section{Fundamentação Teórica}

Das competências que os estudantes do ensino superior do século XXI devem dominar, sobressaem as associadas ao conhecimento sobre o modo como aprendem. A capacidade de conhecer a sua maneira preferida de aprender, como aprendem em diferentes situações pode ser a resposta à necessidade contínua de adaptação a novas realidades (Gallego, 2013).

O conhecimento dos estilos de aprendizagem dos estudantes pode ajudar a melhorar o ensino e a aprendizagem, no sentido dos professores poderem beneficiar desse conhecimento para prepararem os recursos de apoio à aprendizagem, nomeadamente atividades que se adequem às formas de aprendizagem preferidas dos estudantes, tornando a aprendizagem mais atrativa e fácil para os estudantes (Graf \& Kinshuk, 2010). Moreno e Defude (2010) salientam que os estilos 
de aprendizagem referem-se a diferenças individuais entre pessoas quando estão imersas num processo de aprendizagem.

De acordo com Keefe, citado por Alonso, Gallego, e Honey (1999), os estilos de aprendizagem são indicadores cognitivos, afetivos e fisiológicos, relativamente estáveis, do modo como os estudantes percebem, interagem e respondem aos seus ambientes de aprendizagem. Honey e Mumford (1992) consideram quatro estilos de aprendizagem: ativo, reflexivo, teórico e pragmático.

As principais características das pessoas associadas a cada um dos estilos são as seguintes: estilo ativo animador, improvisador, descobridor, ousado e espontâneo; estilo reflexivo - ponderado, consciencioso, recetivo, analítico e exaustivo; estilo teórico - metódico, lógico, objetivo, crítico e estruturado; estilo pragmático - experimentador, prático, direto, eficaz e realista (Alonso, Galego \& Honey, 1999).

De acordo com Bender (2003), quando se atende aos estilos de aprendizagem dos estudantes e o ensino é orientado em função dos estilos de aprendizagem, os estudantes podem tornar-se mais responsáveis e atingir níveis mais elevados de aprendizagem. Gallego e Alonso (2010) defendem que a utilização do conhecimento dos estilos de aprendizagem dos estudantes é essencial na melhoria do processo de ensino e aprendizagem.

Truong (2016) sugere que os estilos de aprendizagem são fontes uteis para o desenvolvimento de elearning e sistemas adaptativos que personalizam os recursos de aprendizagem a as necessidades individuais de aprendizagem dos estudantes.

Tendo em conta a quantidade e potencialidade dos recursos digitais disponíveis para o apoio ao processo de ensino e aprendizagem, constitui um desafio conhecer a utilização que os estudantes fazem destes recursos, e a relação desta utilização com os seus estilos de aprendizagem.

$\mathrm{Na}$ utilização dos recursos digitais, num estudo realizado por Miranda, Morais, Goulão, e Melaré (2012), com uma amostra de 147 sujeitos do ensino superior, concluíram que a maioria dos estudantes do ensino superior utiliza a internet todos os dias, em casa e na Universidade/Trabalho, para procurar informação, fins educativos, comunicar, trabalhar e como forma de entretenimento.

Uma das características individuais que influencia significativamente o modo como os estudantes entendem e participam nos AVA é o seu estilo de aprendizagem. A investigação em estilos de aprendizagem proporciona grande conhecimento para aumentar os resultados académicos, desenho de conteúdos e estratégias de ensino (Küçük, Genç-Kumtepe, \& Tac, 2010).

Num estudo realizado por Graf, Liu e Kinshuk (2010) sobre o comportamento dos estudantes relativo à navegação, num sistema de gestão de aprendizagem num curso online, os autores concluíram que foram identificadas várias diferenças nos padrões de navegação que evidenciam que os estudantes com diferentes estilos de aprendizagem usam diferentes estratégias para aprender e navegar através do curso.
Do resultado de um estudo sobre aspetos do uso de ferramentas digitais, Rodríguez, Lozano, Aradillas e Duque (2011) evidenciam que os estudantes que estiveram integrados em equipas com o mesmo estilo predominante apresentaram perceções favoráveis mais altas, sobre o uso da ferramenta Google Docs e as suas caraterísticas, do que aqueles que estiveram em equipas constituídas por sujeitos de diferentes estilos.

Cela, Fuertes, Alonso e Sánchez (2010) são de opinião que os utilizadores de ferramentas web 2.0 preferem utilizá-las em função do seu estilo de aprendizagem e que a maioria das ferramentas são utilizadas tanto em atividades pessoais como profissionais. Acrescentam, ainda, que as ferramentas web 2.0 que possuem maior preferência de utilização são as de comunicação, blogues, wikis e vídeo.

\section{Metodologia}

O estudo é de natureza quantitativa, as variáveis assumem valores quantitativos e são exploradas relações entre as variáveis. A investigação quantitativa permite testar a relação entre variáveis e obter conclusões pela aplicação de testes e procedimentos estatísticos. Pode-se considerar que um estudo é quantitativo quando se pretende quantificar a variação de um fenómeno, situação, problema ou questão, a informação é obtida através de variáveis essencialmente quantitativas, e a análise da informação orienta-se para avaliar a magnitude da sua variação (Kumar, 2011).

A recolha de dados teve lugar durante o $1 .^{\circ}$ semestre do ano letivo de 2014/2015, e obedeceu a duas técnicas de recolha distintas, conforme os dados estão relacionados com os estilos de aprendizagem, ou com o acesso aos recursos digitais, disponibilizados no ambiente virtual de aprendizagem (AVA). Ambas recorreram a dados armazenados automaticamente no AVA, em função da ação dos sujeitos da amostra.

Os dados relativos à caraterização dos estilos de aprendizagem foram obtidos por questionário, pelo preenchimento do questionário CHAEA, que foi previamente integrado no AVA. Os dados relativos ao número de acessos aos recursos digitais foram registados automaticamente, cada vez que os sujeitos da amostra acederam a cada recurso.

A amostra é constituída pelos estudantes de duas escolas do ensino superior público, uma escola de Educação e a outra de Tecnologia e Gestão, com aproximadamente 3800 estudantes. Desses, preencheram o questionário CHAEA e acederam a recursos digitais 79 sujeitos, os quais constituem a amostra que forneceu dados para este estudo. Dos 79 sujeitos da amostra 77,2\% são do género feminino e $22,8 \%$ do género masculino. Pertencem 50,6\% à escola de Educação e 49,4\% à escola de Tecnologia e Gestão.

As variáveis estudadas estão associadas a estilos de aprendizagem e a recursos digitais. As associadas aos estilos são: ativo, reflexivo, teórico e pragmático, assumindo cada uma delas valores inteiros a variar de zero a 20 pontos. As variáveis associadas aos recursos digitais foram designadas pelos mesmos nomes que estão identificadas no AVA da instituição onde se desenvolveu 
o estudo, são as seguintes: Fóruns, Mensagens, Recursos, Testes Online, e Trabalhos. Cada uma destas variáveis assume valores que variam de zero até ao número máximo de acessos realizado pelo estudante que acedeu maior número de vezes ao recurso.

Do tratamento dos dados destaca-se a análise descritiva, organizados em tabelas e gráficos e a análise inferencial utilizando-se o programa estatístico SPSS, na determinação dos coeficientes de correlação de Spearman entre os pares de variáveis em estudo.

\section{Apresentação e Discussão dos Resultados}

$\mathrm{Na}$ apresentação dos resultados enfatizam-se os aspetos que podem contribuir para responder aos objetivos formulados para a investigação. Apresenta-se a caraterização dos estilos dos sujeitos da amostra e a relação entre os estilos de aprendizagem e a frequência de acesso aos recursos digitais.

\section{Caraterização dos Estilos de Aprendizagem dos Sujeitos da Amostra}

A identificação dos estilos de aprendizagem dos sujeitos que participaram na investigação foi realizada a partir do preenchimento do questionário CHAEA disponível no AVA da instituição onde foi realizado o estudo. O questionário CHAEA (Cuestionario HoneyAlonso de Estilos de Aprendizaje) é constituído por 80 itens breves e dicotómicos, correspondendo 20 a cada estilo de aprendizagem: Ativo, reflexivo, teórico e pragmático. Em termos de pontuação, a resposta a cada item admite a pontuação de um ou zero pontos, consoante está mais ou menos de acordo com o conteúdo do item, respetivamente. A pontuação de cada sujeito em cada estilo é determinada pelo perfil definido pela teoria dos estilos que associa a resposta ao respetivo estilo. $\mathrm{O}$ questionário possui ainda questões sociodemográficas e instruções sobre o seu preenchimento, nomeadamente solicitação para se preencherem todos os itens do questionário, e que a resposta é traduzida com o sinal "+" consoante está mais de acordo do que de desacordo relativamente à afirmação do item, e com o sinal "-_“ quando se está mais de desacordo do que de acordo com a afirmação constante no item em apreciação. As 20 questões relativas a cada estilo estão distribuídas aleatoriamente pelo questionário, no sentido do respondente não poder identificar os itens associados a cada estilo (Miranda \& Morais, 2008).

Na caracterização do nível de preferência dos sujeitos da amostra em cada estilo de aprendizagem foram utilizados os critérios definidos por Alonso, Gallego e Honey (1999), apresentados na Tabela 1.

É assumido que um dos objetivos da identificação dos estilos de aprendizagem dos estudantes consiste em promover o autoconhecimento das características cognitivas, afetivas e fisiológicas que permitam a cada estudante compreender a sua forma de aprender, de interagir e de responder aos ambientes de aprendizagem. Assim, cada estudante poderá beneficiar desse conhecimento para melhorar os seus processos de aprendizagem, conhecendo os indicadores onde está melhor e aqueles onde necessita de melhorar. Neste sentido, cada estudante estará mais preparado para aprender, quanto mais próxima de 20 for a pontuação, em cada um dos estilos de aprendizagem.

Tabela 1

Preferências nos estilos de aprendizagem (Adaptada de Alonso, Gallego \& Honey, 1999, p.114)

\begin{tabular}{lccccc}
\hline \multirow{2}{*}{$\begin{array}{c}\text { Estilos de } \\
\text { Aprendizagem }\end{array}$} & \multicolumn{5}{c}{ Níveis de Preferência } \\
\cline { 2 - 6 } & $\begin{array}{c}\text { Muito } \\
\text { Baixa }\end{array}$ & Baixa & Moderada & Alta & $\begin{array}{c}\text { Muito } \\
\text { Alta }\end{array}$ \\
\hline Ativo & $0-6$ & $7-8$ & $9-12$ & $13-14$ & $15-20$ \\
\hline Reflexivo & $0-10$ & $11-13$ & $14-17$ & $18-19$ & 20 \\
\hline Teórico & $0-6$ & $7-9$ & $10-13$ & $14-15$ & $16-20$ \\
\hline Pragmático & $0-8$ & $9-10$ & $11-13$ & $14-15$ & $16-20$ \\
\hline
\end{tabular}

Para que cada estudante possa identificar o seu nível de preferência em cada estilo de aprendizagem, após o preenchimento do questionário CHAEA, a cada estudante é apresentado no AVA da instituição o seu perfil de aprendizagem em função das pontuações obtidas em cada um dos estilos de aprendizagem.

Para além da vantagem de cada sujeito poder beneficiar por conhecer o seu perfil de aprendizagem, também é relevante para os professores por poderem conhecer o perfil predominante dos estudantes dos cursos que lecionam, pois dessa forma poderão planificar e implementar estratégias de ensino e aprendizagem que vão mais facilmente ao encontro dos interesses e formas de aprender dos estudantes. Considerando a amostra como um todo, pode ser encarada como se de um curso do ensino superior se tratasse. Nesse sentido, atendendo à escala utilizada para cada estilo, de zero a 20 pontos, e para se poderem relacionar as características da amostra em função dos estilos de aprendizagem com a utilização das ferramentas digitais, apresenta-se na Tabela 2 a distribuição dos sujeitos da amostra relativamente aos estilos de aprendizagem e respetivos níveis de preferência. Como cada sujeito pode admitir preferências iguais em mais do que um estilo, de um modo geral, o número de sujeitos em cada nível de preferência é superior ao tamanho da amostra, ou seja, superior a 79 .

Tabela 2

Niveis de preferência nos estilos de aprendizagem $(n=79)$

\begin{tabular}{lcccc}
\hline Níveis & Ativo & Reflexivo & Teórico & Pragmático \\
\cline { 2 - 5 } & $\%$ & $\%$ & $\%$ & $\%$ \\
\hline Muito Alta & 26,6 & 8,9 & 24,1 & 26,6 \\
\hline Alta & 24,1 & 19,0 & 22,8 & 32,9 \\
\hline Moderada & 40,5 & 44,3 & 43,0 & 27,8 \\
\hline Baixa & 6,3 & 19,0 & 7,6 & 10,1 \\
\hline $\begin{array}{l}\text { Muito } \\
\text { Baixa }\end{array}$ & 2,5 & 8,9 & 2,5 & 2,5 \\
\hline
\end{tabular}

Pela observação da Tabela 2 constata-se que o nível de preferência moderada é o que possui maior percentagem de sujeitos nos estilos ativo, reflexivo e teórico, enquanto no estilo pragmático a preferência com maior percentagem de sujeitos é a preferência alta. Com níveis de preferência alta ou muito alta surgem $51,7 \%$, no estilo 
ativo, $27,9 \%$ no estilo reflexivo, $46,9 \%$ no estilo teórico e $59,5 \%$ no estilo pragmático. Com níveis de preferência baixa ou muito baixa, existem $8,8 \%$ no estilo ativo, $27,9 \%$ no estilo reflexivo, $10,1 \%$ no estilo teórico e $12,6 \%$ no estilo pragmático. Estes dados permitem inferir que os sujeitos da amostra necessitam de melhorar o perfil associado ao estilo reflexivo, pois é no estilo reflexivo onde se verifica menor percentagem se sujeitos com níveis de preferência alta ou muito alta.

Apresenta-se na Tabela 3, uma síntese descritiva dos dados sobre as pontuações obtidas pelos sujeitos da amostra em cada um dos estilos de aprendizagem.

Tabela 3

Estatísticas das pontuações obtidas nos estilos de aprendizagem $(n=79)$

\begin{tabular}{lccccc}
\hline $\begin{array}{l}\text { Estilos de } \\
\text { aprendizagem }\end{array}$ & Mín & Máx. & Média & Mediana & Moda \\
\hline Ativo & 5 & 18 & 12,4 & 13 & 12 \\
\hline Reflexivo & 7 & 20 & 15,2 & 16 & 16 \\
\hline Teórico & 6 & 20 & 13,4 & 13 & 13 \\
\hline Pragmático & 8 & 20 & 13,8 & 14 & 14 \\
\hline
\end{tabular}

Pela Tabela 3, constata-se que o máximo das pontuações foi atingido nos estilos reflexivo, teórico, e pragmático, não tendo sido atingido no estilo ativo, também foi neste estilo que se verificou o mínimo das pontuações e a média das pontuações mais baixa. Por outro lado, foi no estilo reflexivo que se verificaram a média $(15,2)$ e a moda (16) mais elevadas das pontuações.

\section{Relação Entre os Estilos de Aprendizagem e o Acesso a Recursos Digitais}

No sentido de melhorar as estratégias de ensino e aprendizagem, tendo em conta os estilos de aprendizagem dos estudantes foi dada particular ênfase à relação dos estilos predominantes dos estudantes com a frequência de acesso às ferramentas do AVA, nomeadamente Fóruns, Mensagens, Recursos, Testes Online e Trabalhos.

$\mathrm{Na}$ Tabela 4, apresenta-se uma síntese dos dados relativos ao acesso dos sujeitos da amostra aos recursos digitais, disponíveis no AVA.

Tabela 4:

Acesso às ferramentas do AVA

\begin{tabular}{lccccc}
\hline \multirow{2}{*}{ Ferramentas } & \multicolumn{5}{c}{ Acessos (3732) } \\
\cline { 2 - 6 } & N. $^{\mathbf{0}}$ & $\%$ & Máximo & Mínimo & Média \\
\hline Fóruns & 154 & 4 & 40 & 0 & 1,9 \\
\hline Mensagens & 232 & 6 & 12 & 0 & 2,9 \\
\hline Recursos & 3297 & 88 & 251 & 0 & 41,7 \\
\hline Testes & 27 & 1 & 5 & 0 & 0,3 \\
Online & & & & & \\
\hline Trabalhos & 22 & 1 & 6 & 0 & 0,3 \\
\hline
\end{tabular}

Os resultados apresentados na Tabela 4, são evidenciados na Figura 2.

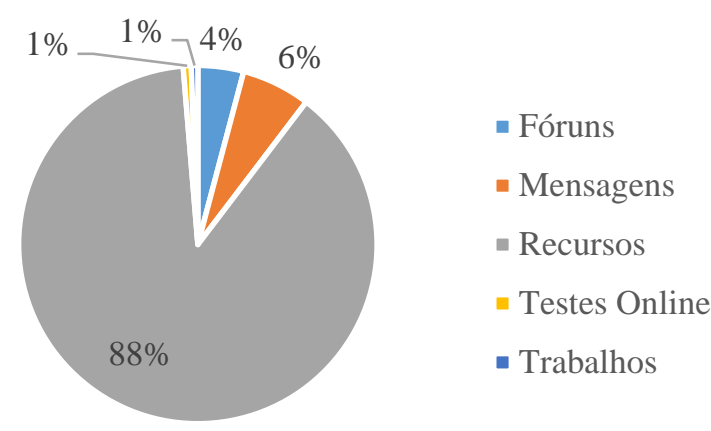

Figura 2: Acesso às ferramentas ao AVA (3732 acessos)

Pela observação da Tabela 4 e da Figura 2 constata-se que a ferramenta com maior número de acessos, é a ferramenta recursos, sendo praticamente irrelevante o uso que os estudantes fazem das outras ferramentas. Este facto implica uma reflexão profunda sobre o uso que os estudantes fazem dos recursos digitais. Pois, não basta têlos disponíveis, é fundamental incentivar os estudantes à sua utilização, sendo essencial levar os estudantes a admitirem que a utilização dos recursos digitais pode melhorar o seu desempenho académico.

Depois da identificação da frequência dos acessos às ferramentas digitais, procurou-se compreender se esse acesso é influenciado pelo perfil dos estudantes em termos de estilos de aprendizagem, procurando identificar os coeficientes de correlação entre as variáveis.

Considerando que as variáveis relativas aos estilos de aprendizagem, pontuações obtidas por cada sujeito nos estilos ativo, reflexivo, teórico e pragmático, assim como as variáveis associadas à frequência de acesso aos recursos digitais, associadas ao número de vezes que acederam a Fóruns, Mensagens, Recursos, Testes Online, e Trabalhos são variáveis quantitativas, de acordo com Pereira (2004) o grau de associação entre as variáveis pode ser determinado utilizando o coeficiente de correlação de Pearson quando a distribuição das variáveis é normal, ou através do coeficiente de correlação de Spearman, quando a distribuição das variáveis não é normal.

Para averiguar se a distribuição das variáveis é normal, utilizou-se o teste de Kolmogoronov- Smirnov (K-S) (Maroco, 2010). Pela aplicação do teste K-S, através do SPSS, constatou-se que apenas as variáveis associadas aos estilos de aprendizagem ativo, reflexivo, teórico e pragmático, admitem distribuição normal e que não admitem distribuição normal as variáveis Fóruns, Mensagens, Recursos, Testes Online, e Trabalhos. Pelo exposto, as distribuições das variáveis em estudo não satisfazem as condições de aplicabilidade da correlação de Pearson, implicando a opção pela determinação dos coeficientes de correlação de Spearman (Pereira, 2004).

Dos resultados da determinação dos coeficientes de correlação, e tendo em conta os valores encontrados para os coeficientes de correlação resultantes da aplicação do teste de Spearman, apenas se evidencia que a correlação é positiva e fraca $(0,229)$, com um nível de significância inferior a 0,05 , entre as variáveis estilo teórico e 
mensagens. Em todas as outras situações, entre qualquer um dos pares de variáveis considerados não existe correlação significativa com um nível de significância inferior a 0,05 . Assim, pelos dados obtidos não se pode afirmar que os estilos de aprendizagem dos sujeitos da amostra influenciam o número de acessos dos estudantes aos recursos digitais.

\section{Conclusões}

O estudo decorreu com uma amostra de 79 estudantes do ensino superior público português, com o qual se pretendeu averiguar as caraterísticas dos sujeitos da amostra relativamente aos estilos de aprendizagem, utilizando a classificação dos estilos proposta por HoneyAlonso, ou seja, ativo, reflexivo, teórico e pragmático, admitindo-se para cada estilo os níveis de preferência muito baixa, baixa, moderada, alta e muito alta.

Numa escala de zero a vinte pontos para cada estilo, as médias das pontuações variaram de 12,4 no estilo ativo a 15,2 no estilo reflexivo, sendo de 13,4 no estilo teórico e 13,8 no estilo pragmático. Os dados não permitiram construir grupos independentes com caraterísticas vincadamente diferentes em função dos estilos dos sujeitos da amostra.

Relativamente às ferramentas utilizadas Fóruns, Mensagens, Recursos, Testes Online e Trabalhos, no AVA da instituição, durante um semestre, verificou-se que o número de acessos da amostra variou de 22 na ferramenta Trabalhos a 3297 na ferramenta Recursos, correspondendo o número de acessos à ferramenta Recursos $88 \%$ da totalidade dos acessos. Assim, pode-se concluir que a ferramenta que é utilizada com frequência bastante elevada é a ferramenta Recursos.

Sobre a relação entre o número de acessos a recursos digitais e os estilos de aprendizagem, utilizou-se o SPSS, e os coeficientes de correlação de Spearman. Não foram encontradas evidências que permitam afirmar que os estilos de aprendizagem influenciam significativamente o número de acessos dos estudantes aos recursos digitais.

\section{Referencias}

Alonso, C., Gallego, D., \& Honey, P. (1999). Los estilos de aprendizage: Procedimientos de diagnóstico y mejora (4th ed.). Bilbao: Ediciones Mensajero.

Bender, T. (2003). Discussion-based online teaching to enhance student learning: Theory, practice and assessment. Sterling, Virginia: Stylus Publishing, LLC.

Cela, K., Fuertes, W., Alonso, C., \& Sánchez, F. (2010). Evaluación de herramientas web 2.0, estilos de aprendizaje y su aplicación en el ámbito educativo. Revista Estilos de Aprendizaje, 5(5), 117-134.

Gallego, D. \& Alonso, C. (2010). Estilos de Aprendizaje. In J. Cué, J. Rineón, e M. Velázques Editors, IV Congreso Mundial de Estilos de Aprendizaje, pp. 2448. México: México.

Gallego, D. (2013). Ya he diagnosticado el estilo de aprendizaje de mis alumnos y ahora qué hago?, Revista de Estilos de Aprendizaje, 11(12), 1-15.

Graf, S. \& Kinshuk, T. (2010). Analysis of learners' navigational behaviour and their learning styles in an online course, Journal of Computer Assisted Learning, 116-131.

Graf, S., Liu, T., \& Kinshuk, T. (2010). Analysis of learners' navigational behaviour and their learning styles in an online course. Journal of Computer Assisted Learning, 26, 116-131.

Honey, P. \& Mumford, A. (1992). The manual of learning styles. Maidenhead: Peter Honey.

Küçük, M., Genç-Kumtepe, E. \& Tac, D. (2010). Support services and learning styles influencing interaction in asynchronous online discussions. Educational Media International, 47(1), March 2010, 39-56.

Kumar, R. (2011). Research methodology: A step-bystep guide for beginners. London: SAGE Publication Ltd.

Maroco, J. (2010). Análise estatística: Com utilização do SPSS (3. ${ }^{a}$ Ed.). Lisboa: Edições Sílabo,

Miranda, L. \& Morais, C. (2008). Estilos de aprendizagem: O questionário CHAEA adaptado para língua portuguesa. Revista de Estilos de Aprendizagem, 1 (1), 66-87.

Miranda, L., Morais, C., Goulão, F., \& Melaré, D. (2012). Estilos de aprendizagem: Uso do virtual por alunos do ensino superior. Revista de Estilos de Aprendizaje, 1(10), Octubre de 2012, 184-195.

Moreno, J. \& Defude, B. (2010). Learning styles and teaching strategies to improve the SCORM Learning Objects Quality. In Steimle, Jürgen (ed.) Proceedings of the 10th IEEE International Conference on Advanced Learning Technologies ICALT10, pp. 414416, July 5-7, Sousse, Tunisia.

Pereira, A. (2004). Guia prático de utilização do SPSS. Lisboa: Edições Sílabo, 2004.

Rodríguez, A., Lozano, D., Aradillas, A., \& Duque, E. (2011). Uso de google docs como herramienta de construcción colaborativa tomando en cuenta los estilos de aprendizaje. Revista Estilos de Aprendizaje, 8 (8), 23-29.

Truong, H. (2016). Integrating learning styles and adaptive e-learning system: Current developments, problems and opportunities, Computers in Human Behavior, 55 , 1185-1193. 